\title{
Motivaciones de compra de productos de consumo habitual en hogares de la ciudad de México
}

\section{Purchasing motivations of customary consumption products in Mexico city households}

\author{
Laura Estela Fischer de la Vega (México) \\ Ifischer@fca.unam.mx \\ Universidad Nacional Autónoma de México \\ Doctora en Ciencias Sociales y Administrativas
}

Jorge Ángel Espejo Callado (México)
jorgeaespejocallado@gmail.com
Universidad Autónoma del Estado de Morelos
Maestría en Administración de Empresas

\section{Resumen}

Con el propósito de identificar los motivos de naturaleza intencionada y determinista que subyacen a la compra repetitiva, la duplicidad y sustitución de marca, la cuota de cartera y la lealtad

\section{Summary}

In order to identify the intentional and deterministic natural motives that lay under repetitive shopping, brand duplication and substitution, porffolio share and shared loyalty, a longitudinal /

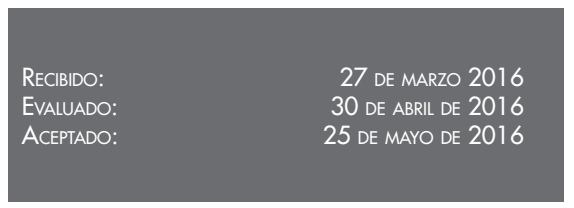

PARA CITAR ESTE ARTículo / TO CITE THIS ARTIClE

Fischer de la Vega, L. E. \& Espejo Callado J. Á. (2016). Motivaciones de compra de productos de consumo habitual en hogares de la Ciudad de México, Bogotá: Editorial Politécnico Grancolombiano, Poliantea, 12(23), pág 55-73 
compartida, se realizó una investigación de naturaleza longitudinal/transversalcuantitativa/cualitativa en donde, a lo largo de 12 semanas, se analizó la tendencia sistemática de repetición de compra de una muestra de 60 hogares mexicanos radicados en la Ciudad de México. Al concluir el levantamiento de la última ola de datos y, teniendo siempre presente que los integrantes de la familia juegan un papel definitorio en los patrones de consumo del hogar, se realizó un sesión de grupo en cada hogar, encontrándose que los factores de motivación de compra en productos de consumo habitual no se identifican en forma exclusiva con alguno de los enfoques, escuelas o teorías del comportamiento de compra, sin embargo, las escuelas conductista, cognitiva y económica son las que más se identifican en las decisiones de compra de productos de consumo recurrente.

Palabras clave: motivación de compra; compra habitual; patrones de consumo; compra repetida. transversal-quantitative / qualitative research was conducted, and over a 12 weeks period, the repetitive purchase systematic trend of a sample of 60 Mexico City households was analyzed. Concluding the last wave of data, and keeping in mind that every family member plays a defining role in household consumption patterns, a group session was carried out in each household, finding that motivation purchase factors in customary products are not uniquely identified with any approach, school or purchasing behavioral theory. However, behavioral, cognitive and economic schools are the most identified in purchasing decisions of customary products.

Keywords: purchase motivation; customary purchase; consuming patterns; repeated purchase. 


\section{Introducción}

A través de los años, mercadólogos, sociólogos, psicólogos, economistas y antropólogos se han mostrado profundamente interesados en precisar las motivaciones que llevan a un consumidor a realizar sus compras; en la actualidad, en un mundo tan competido y saturado de productos y marcas de características similares, que buscan satisfacer las mismas necesidades de un consumidor en permanente evolución, el cuestionamiento sigue siendo vigente. Académicos, investigadores y practicantes no se sienten satisfechos y se siguen preguntando ¿qué es lo que hace que un consumidor se incline por una marca en lugar de otra?; eventualmente, los esfuerzos por comprender necesidades, deseos y expectativas, particularmente durante el periodo de transición del consumidor (de situación actual a la situación deseada) dan frutos, permitiendo a las empresas identificar, clasificar y proponer los motivos o fuerzas de impulso irresistibles que detonan en el consumidor la compra del bien o servicio de su marca; en este documento se recuperan y ejemplifican los diferentes enfoques motivacionales que explican por qué compra el consumidor, y queda claro que es trascendente conocer su comportamiento con el fin de seleccionar la estrategia de mercadotecnia correcta. Esta no es una tarea fácil de lograr, dado que el comportamiento del consumidor está en constantemente cambio; entender por qué los clientes realizan compras, por qué seleccionan una marca en lugar de otra, o por qué eligen un vendedor en lugar de otro implica conocer sus motivaciones. 


\section{Marco teórico}

Diferentes teorías se han adoptado en los últimos dos siglos para abordar el tema del consumo, esta heterogeneidad de aproximaciones explica la decisión de compra desde la perspectiva de los sistemas productivos, el juego de la oferta y la demanda, las necesidades humanas, las relaciones sociales y el individualismo (Usín, 2013); investigadores y académicos han sugerido una clasificación con diversas tipologías, sin embargo, encontramos que son nueve los enfoques principales: Hombre Económico, Psicodinámico, Conductista, Cognitivo, Humanista, Social, Estructuralismo, Valores y Postmodernismo (Petrauskaite, 2014), cada uno de estos nueve enfoques postulan modelos alternos, y hacen hincapié en la necesidad de examinar muy diferentes variables (Foxall, 1990).

Desde la perspectiva del hombre económico, los consumidores tienden a maximizar sus satisfacciones, se les considera decisores totalmente racionales y auto-interesados, es decir, expresan un alto interés en la utilización del producto con un mínimo esfuerzo. Cuando un consumidor tiene este enfoque siempre considera todas las opciones disponibles, las valora en un orden específico, y selecciona el producto que arroje el valor óptimo (Schiffman, 2007); según Simon (1997), sin la recopilación de información adecuada, la decisión "perfecta" del consumidor no podría formularse.

El enfoque psicodinámico dentro de la psicología se atribuye al trabajo de Sigmund Freud (18561939) (Stewart, 1994). Esta visión plantea que el comportamiento del consumidor es impulsado subjetiva o inconscientemente por factores biológicos llamados instintos, como los sentimientos que actúan fuera del pensamiento consciente, y no por conductores de naturaleza objetiva como el conocimiento; el enfoque supone que la mayoría de la características del comportamiento del consumidor ya se formaron en la infancia, por ejemplo, las personas tienden a elegir una bebida dulce en lugar de una amarga (McLeod, 2013).

Según Bray (2008), el enfoque conductista es una familia de filosofías que describe el comportamiento de compra como una respuesta a estímulos externos e internos, y es explicado tanto por la teoría clásica del acondicionamiento de Ivan Pavlov (1849-1936) como por la teoría del condicionamiento operante 
de Burrhus Skinner (1904-1990), quien afirma que los consumidores cuando compran tienden a combinar los dos estímulos; esta teoría tiene importantes implicaciones para las situaciones en las cuales deseamos facilitar cambios en lo que la gente conoce o hace; para Ertmer y Newby el conductismo se asemeja al aprendizaje por los cambios en la conducta observable, bien sea respecto a la forma o a la frecuencia de esas conductas, la satisfacción en la compra hará que esta se vuelva repetida sin existir una reflexión hacia lo comprado (Ertmer, 1993).

El enfoque cognitivo convierte las experiencias en cognición personal, el consumidor es percibido como un procesador de información que se encuentra en permanente búsqueda de estímulos ambientales y sociales para convertirlos en conocimientos útiles para la decisión de compra; el enfoque propone que la experiencia de compra adquirida por el consumidor influiría en sus futuras decisiones, esto significa que un consumidor recopila información acerca de una marca específica con la que puede o no estar familiarizado, y extrae los datos específicos que le servirán de referencia para decidir futuras compras (Stewart,
1994); las teorías cognitivas enfatizan la adquisición del conocimiento y estructuras mentales internas $y$, como tales, están más cerca del extremo racionalista (Bower, 1981); el enfoque cognitivo reconoce la postura activa del consumidor como tomador de decisiones racionales sobre la base de la información recogida, así como el papel activo del medio ambiente y la experiencia social como proveedores de estímulos ambientales y sociales (Stewart, 1994).

El enfoque humanista se centra en las emociones de los consumidores en el momento de la acción de compra. Los investigadores de esta perspectiva del consumo están más interesados en explorar conceptos introspectivos del consumidor individual y menos en describir procesos genéricos (Stewart, 1994); Hoyer y MacInnis aseguran que de esta forma las estrategias de mercadotecnia estarían mejor adaptadas al mercado (Hoyer, 2010).

El enfoque social-cultural se le acredita a Thorstein Veblen (1899), por ser el primero en formular las "teorías culturales del consumo", influenciado no solo por su formación económica sino también sociológica (Usín, 2013), (Fischer, 2011); Veblen 
afirmó que el individuo es esencialmente un animal social, y que sus necesidades y comportamientos están en gran parte influenciados por los miembros de los grupos a los que pertenece; sostuvo que la gente tiende a formar parte de una sociedad a pesar de sus diferencias y gustos muy personales: cultura, subcultura, clases sociales, grupos de referencia y familia agrupan diversos factores que influyen en el comportamiento como comprador. Con el paso del tiempo, la cultura de consumo ha venido incorporando nuevas interpretaciones como el concepto de "ir de compras (shopping)", y reinterpreta la relación del sujeto con los bienes y servicios (cultura material), donde los significados sociales cobran fuerza y sentido (Veblen, 1899, citado por Usín, 2013).

Sheth, Newman y Gross (Sheth, 1991), afirman que el estructuralismo es una teoría que enfatiza el papel desempeñado por los signos y símbolos, traslada los mitos y los rituales de las sociedades antiguas a la sociedad moderna; según esta teoría, el individuo no solo vive en un universo físico, sino que también habita en un universo simbólico, compuesto por el arte, la lengua, las religiones, los mitos, etc.; el papel de los signos y símbolos resulta trascendente en el proceso de consumo y se manifiesta mediante un mecanismo dominante de nuestra sociedad llamado publicidad; para los autores, la intención de comprar un producto implica para el consumidor tomar decisiones que no solo están conducidas por sus experiencias y expectativas, sino también por sus valores; es entonces la teoría de los valores de consumo la que explica por qué los consumidores eligen y compran una marca de producto o servicio y no otras.

Las nuevas concepciones sobre producción, consumo, clase social, etc., dan paso a una nueva forma de entender la sociedad a la que se le viene denominando postmoderna y que se caracteriza, entre otras cosas, por los mercados cada vez más segmentados y consumos diferenciados, individuales, virtuales y globales; en esta época, la sociedad mexicana se empieza a describir en función a estilos de vida tribales, grupos similares en torno al consumo, sin conciencia de clase, pero que ansían pertenecer a un mismo grupo que les permita alcanzar un determinado estatus y construir una identidad social a través del consumo (Usín, 2013), (Fischer, 2011); es 
dentro de esta nueva corriente de pensamiento que Fischer y Espejo (2015) sintetizaron las motivaciones de compra en tres dimensiones principales: necesidades innatas o absolutas, necesidades relativas y esfuerzos mercadológicos, en un estudio realizado en la República de México en el que participaron 2.400 estudiantes universitarios con estilos de vida tribal. Los investigadores encontraron que existe una relación (influencia) importante entre los esfuerzos de mercadotecnia desarrollados por las empresas y la decisión de compra, incluso superior a la influencia que pudieran ejercer las necesidades innatas y sociales; sin embargo, para atraer y retener a los consumidores, las estrategias deben construirse de manera equilibrada, esto es, integrando también los factores de motivación innata y social; este hallazgo sugirió una nueva línea de investigación ahora relacionada con las razones que motivan la compra de productos de consumo recurrente en los hogares.

\section{Metodología \\ Objetivo}

Identificar los motivos de naturaleza intencionada y determinista que subyacen a la compra repetitiva, la duplicidad y sustitución de marca, la cuota de cartera y la lealtad compartida.

\section{Hipótesis}

H1. A pesar de que los productos de consumo recurrente siguen una tendencia sistemática de compra, los motivos de naturaleza intencionada y determinista que subyacen a la compra repetitiva, la duplicidad y sustitución de marca, la cuota de cartera y la lealtad compartida, no se identifican en forma exclusiva con alguno de los enfoques, escuelas o teorías de la motivación de compra.

\section{Diseño de la investigación}
A. Estudio longitudinal/transversal, cuantitativo/cualitativo
B. Definición del universo
- Hogares privados en la Ciu- dad de México: 2.453.031 (INEGI, 2011)
- Hogares en los NSE AB, C+, C, C- en la Ciudad de México: 1.358.979 (Romo, 2009) 
c. Universo de estudio

- La investigación utilizó una muestra de 60 hogares residentes en la zona sur del área metropolitana de la Ciudad de México, ver tabla 1.
- Las principales características de los hogares panelistas participantes, y fundamentales para dimensionar los hallazgos cualitativos se muestran en la tabla 2.

Tabla 1. Conformación de la muestra de hogares.

\begin{tabular}{|c|c|c|c|c|}
\hline Hogares & $\mathrm{AB}$ & $\mathrm{C}+$ & $\mathrm{C}$ & $\mathrm{C}$ \\
\hline Participantes & 4 & 16 & 13 & 27 \\
\hline Proporión & $6,3 \%$ & $26,3 \%$ & $20,9 \%$ & $45,0 \%$ \\
\hline AMAl1 Nacional & $7,6 \%$ & $13,7 \%$ & $17,0 \%$ & $17,1 \%$ \\
\hline Hogares DF & 186.430 & 336.065 & 417.015 & 419.468 \\
\hline
\end{tabular}

Fuente: Elaboración propia.

\section{a) Determinación de la muestra}

$\varnothing \quad$ Muestreo mixto, por conveniencia y rotativo

b) Método de recolección de datos: Cuantitativo: panel de hogar Cualitativo: sesión de grupos

c) Instrumento de medición: Cuantitativo: diario de compras (anexos 1 y 2)

Cualitativo: guía de tópicos (anexo 3)

Tabla 2. Perfil general de las familias panelistas.

Integrantes en el hogar (promedio)

Hogares de núcleo básico

Hogares de núcleo extendido

Hogares comandados por figura materna

Hogares comandados por figura paterna

Edad promedio de la madre

Edad promedio del padre

Edad promedio de los hijos

Familiares conviviendo con el núcleo básico (prom.) 2.3

Descendientes de sexo masculino

Descendientes de sexo femenino
4.3

$39(65 \%)$

21

$26(43 \%)$

34

47.5

53.8

18.8

29

$35(55 \%)$

Fuente: Elaboración propia. 


\section{Hallazgos estudio cuantitativo}

Las tablas 3 y 4 contienen un resumen de los parámetros observados en la compra de 75 marcas de consumo recurrente de las categorías alimentos, higiene, belleza y uso doméstico pertenecientes al canasto de productos Nielsen (2014).

Tabla 3. Parámetros descriptivos de una semana típica dentro de un periodo de 12 semanas ${ }^{1}$

\begin{tabular}{|c|c|c|c|c|c|c|}
\hline \multicolumn{2}{|r|}{ Parámetros } & \multirow{2}{*}{$\begin{array}{c}\text { Cereales } \\
7\end{array}$} & \multirow{2}{*}{$\frac{\text { Yogurt }}{14}$} & \multirow{2}{*}{$\begin{array}{c}\text { Papel Higiénico } \\
20 \\
\end{array}$} & \multirow{2}{*}{$\begin{array}{c}\text { Shampoo } \\
29 \\
\end{array}$} & \multirow{2}{*}{$\begin{array}{c}\text { Atún enlatado } \\
5 \\
\end{array}$} \\
\hline 1 & \# de marcas monitoreadas & & & & & \\
\hline 2 & \# de familias panelistas & 60 & 60 & 60 & 60 & 60 \\
\hline 3 & \# de visitas de compra promedio & 18.3 & 28.1 & 10.9 & 4.7 & 24.2 \\
\hline 4 & \# de familias compradoras promedio & 9.1 & 10.9 & 4.6 & 3.1 & 10.8 \\
\hline 5 & Penetración promedio & $15.2 \%$ & $18.1 \%$ & $7.7 \%$ & $5.1 \%$ & $18.0 \%$ \\
\hline 6 & Participación promedio & $14.3 \%$ & $7.1 \%$ & $5.0 \%$ & $3.4 \%$ & $20.0 \%$ \\
\hline 7 & Frecuencia media de compra & 2.0 & 2.6 & 2.4 & 1.5 & 2.2 \\
\hline 8 & Hogares repetidores promedio & 4.6 & 6.9 & 6.7 & 6.7 & 6.6 \\
\hline 9 & $\%$ promedio de hogares repetidores & $7.6 \%$ & $11.4 \%$ & $11.2 \%$ & $11.2 \%$ & $11.0 \%$ \\
\hline 10 & Duplicidad de marca promedio & $15.6 \%$ & $19.5 \%$ & $7.6 \%$ & $7.2 \%$ & $19.1 \%$ \\
\hline 11 & Coeficiente de Duplicidad promedio & 1.03 & 1.08 & 0.99 & 0.86 & 0.66 \\
\hline 12 & Duplicidad teórica (pronóstico) promedio & $14.6 \%$ & $7.7 \%$ & $5.0 \%$ & $3.0 \%$ & $13.2 \%$ \\
\hline 13 & Duplicidad promedio entre pares de marcas & 1.89 & 1.34 & 1.32 & 1.67 & 0.79 \\
\hline 14 & \% de compradores únicos & $46.7 \%$ & $18.3 \%$ & $41.7 \%$ & $40.0 \%$ & $36.7 \%$ \\
\hline 15 & $\%$ de compradores leales a 2 marcas & $25.0 \%$ & $31.7 \%$ & $26.7 \%$ & $16.7 \%$ & $26.7 \%$ \\
\hline 16 & $\%$ de compradores leales a 3 marcas & $3.3 \%$ & $15.0 \%$ & $11.7 \%$ & $10.0 \%$ & $0.0 \%$ \\
\hline 17 & $\%$ de compradores leales a 4 marcas & $0.0 \%$ & $23.3 \%$ & $1.7 \%$ & $5.0 \%$ & $0.0 \%$ \\
\hline 18 & $\%$ de compradores leales a 5 marcas & $0.0 \%$ & $6.7 \%$ & $3.3 \%$ & $5.0 \%$ & $0.0 \%$ \\
\hline 19 & $\%$ de no Compradores & $25.0 \%$ & $5.0 \%$ & $15.0 \%$ & $23.3 \%$ & $36.7 \%$ \\
\hline 20 & Cuota de cartera (\% del valor del ticket) & $2.85 \%$ & $4.75 \%$ & $4.23 \%$ & $2.92 \%$ & $2.84 \%$ \\
\hline
\end{tabular}

Fuente: Elaboración propia.

Tabla 4. Parámetros estocásticos ${ }^{2}$

\begin{tabular}{|c|c|c|c|c|c|c|c|c|c|c|}
\hline & \multicolumn{4}{|c|}{ Penetración para una semana típica } & \multicolumn{3}{|c|}{ Frecuencia media de compra c/4 sem } & \multicolumn{3}{|c|}{ Proporción de repetidores $\mathrm{c} / \mathbf{1 2}$ semanas } \\
\hline Categorías & $b_{T}$ (NBD) & $b_{T}(L S D)$ & $b_{T}(E A)$ & MKV & $w$ (NBD) & $w($ LSD) & $w(E A)$ & $b_{R}$ (NBD) & $b_{R}$ (LSD) & $b_{R}$ (EA) \\
\hline Cereal & $2.3 \%$ & $2.2 \%$ & $2.2 \%$ & $1.3 \%$ & 1.2 & 0.9 & 1.2 & $40.2 \%$ & $37.7 \%$ & $36.2 \%$ \\
\hline Yogurt & $3.4 \%$ & $3.2 \%$ & $3.2 \%$ & $1.5 \%$ & 1.4 & 1.0 & 1.4 & $43.2 \%$ & $40.4 \%$ & $40.0 \%$ \\
\hline P. Higiénico & $1.3 \%$ & $1.1 \%$ & $1.2 \%$ & $0.6 \%$ & 1.5 & 1.0 & 1.5 & $33.4 \%$ & $32.7 \%$ & $32.4 \%$ \\
\hline Shampoo & $0.6 \%$ & $0.5 \%$ & $0.6 \%$ & $0.4 \%$ & 1.1 & 0.5 & 1.1 & $19.0 \%$ & $18.5 \%$ & $17.5 \%$ \\
\hline Atún & $3.0 \%$ & $2.9 \%$ & $2.9 \%$ & $1.5 \%$ & 1.3 & 0.9 & 1.3 & $50.0 \%$ & $35.9 \%$ & $35.2 \%$ \\
\hline Promedio & $2.1 \%$ & $2.0 \%$ & $2.0 \%$ & $1.1 \%$ & 1.3 & 0.9 & 1.3 & $37.2 \%$ & $33.0 \%$ & $32.3 \%$ \\
\hline
\end{tabular}

Fuente: Elaboración propia.

1 Mayor detalle puede ser consultado en el primer reporte de esta investigación titulado: "Comportamientos de lealtad a la marca en hogares de la Ciudad de México" (Fischer, L., Espejo, J., 2016a).

2 Mayor detalle puede ser consultado en el segundo reporte de esta investigación titulado: "Generalizaciones en la compra repetida, duplicidad de marca, sustitución de marca, lealtad compartida entre marcas y doble riesgo en hogares de la Ciudad de México" (Fischer, L., Espejo, J, 2016b) 
Notaciones:

$\begin{array}{ll}\boldsymbol{b}_{T} \boldsymbol{b}_{T} & \text { Penetración Teórica } \\ \boldsymbol{W} \boldsymbol{W} & \text { Frecuencia Media de } \\ & \text { Compra } \\ \boldsymbol{b}_{\boldsymbol{R}} \boldsymbol{b}_{R} & \begin{array}{l}\text { Proporción de } \\ \text { Repetidores }\end{array} \\ \boldsymbol{N B D N B D} & \text { Modelo Distribución } \\ & \text { Binomial Negativa } \\ & \text { (NBD, por sus siglas } \\ & \text { en inglés) }\end{array}$

LSDLSD Modelo Distribución Logarítmica (LSD, por sus siglas en inglés)

EAEA Modelo de Expresiones Aproximadas

MKVMKV Modelo Cadenas de Markov

Los parámetros presentados en la tabla 4 sugieren la siguiente interpretación desde la perspectiva del modelo NBD (siendo similar para los otros tres modelos utilizados): dado que el $31,7 \%$ de la muestra compró la misma marca en promedio 2,21 veces (parámetros observados en el período de doce semanas), la estimación es que alrededor del $2,3 \%$ de la muestra debe comprar esa marca en el período de una semana típica; que la frecuencia media con la cual los compradores la adquirirán cada cuatro semanas es de alrededor de 1,2 veces; y que la proporción de compradores que repetirán la compra en un mismo periodo de doce semanas es alrededor del $40,2 \%$, esta última proporción también puede entenderse como índice de lealtad a la marca.

\section{Hallazgos estudio cualitativo ¿Por qué en este hogar se consumen las mismas marcas?}

En el tema de la repetición de marcas, las declaraciones de las familias panelistas permiten concluir que su canasta de productos de consumo recurrente está dividida en dos grupos: (a) un grupo primario integrado por marcas que cubren convincentemente requerimientos "básicos" (gustos, necesidades, preferencias, costumbres, hábitos, expectativas); esta es la principal justificación a la compra repetida, las marcas de este grupo se seguirán comprando aun cuando los precios aumenten, el grupo primario de marcas es estable en el tiempo pero no perenne; (b) el segundo grupo lo denominamos secundario precisamente porque cubren requerimientos secundarios de las familias, se trata de marcas elegidas para satisfacer gustos, necesidades y costumbres no cotidianas; el grupo secundario de 
marcas es más volátil, las marcas están sujetas a cambio por cualquier motivo; sin embargo, se puede asegurar que las marcas que forman estos dos grupos gozan de lealtad; prácticamente el ciento por ciento de los hogares panelistas (solo hay una excepción) reportó marcas de compra repetitiva.

Los principales argumentos expresados para justificar la compra repetida de marcas son los siguientes:

- "Es la misma marca que usaban en casa cuando estaba soltera" (61,7\% de los hogares); otros motivadores expresados son: tradición / costumbre / herencia / nostalgia / inercia / experiencia previa (enfoques conductista y humanista).

- "Siempre que uso esta marca obtengo los resultados que espero" (31,7\% de los hogares); otros motivadores expresados son: experiencia acumulada favorable / satisfacción acumulada (enfoques conductista, cognitivo y humanista).

- "Para nuestra familia es la marca que mejor funciona" (26,7\% de los hogares); otros motivadores expresados son: funcionalidad
/ estilo de vida / atributos / beneficios / es lo mejor entre las marcas disponibles (enfoques económico, conductista, cognitivo y socio-cultural).

- "Estas marcas me dan seguridad porque las conozco y sé que funcionan para nuestra familia" (16,7\% de los hogares); otros motivadores expresados son: seguridad / confiabilidad / consistencia (enfoques económico y cognitivo).

\section{¿Por qué en este hogar se llega a cambiar de marca?}

Se observó que son los padres de familia o familiares adultos los más proclives a adquirir las mismas marcas en cada ocasión de compra. Cuando la tarea de compras es delegada a los hijos es frecuente el cambio de marca; con el tiempo la compra recurrente de una marca se convierte en lealtad, pero no es incondicional, los cambios de marca se pueden presentar, por eso es que los grupos de marcas observados y comentados anteriormente se consideran temporales.

Los principales argumentos que justifican el cambio de marca son los siguientes: 
- "Cambiamos de marca cuando se modifica en forma importante el precio, la calidad o la cantidad" (40\% de los hogares); otro motivador expresado es: malas experiencias (enfoque económico, cognitivo y estructuralista).

- "En nuestro hogar no estamos casados con ninguna marca, siempre estamos al pendiente de nuevos productos o mejoras en los existentes" (33,3\% de los hogares); otros motivadores expresados son: recomendaciones de familiares y amistades / publici$\mathrm{dad} /$ esfuerzos promocionales de la competencia / curiosidad / variedad / cambios en la etapa del ciclo de vida familiar (enfoques económico, conductista, cognitivo, social-cultural y estructuralista).

- "Compro otra marca cuando no encuentro la que acostumbramos, pero solo en una situación de emergencia" (20\% de los hogares), el motivador central aquí es el desabasto (enfoques conductista y estructuralista).

- "Cambiamos de marca cuando hay un festejo en casa o recibimos visitas que sabemos les gusta otra marca" (15\% de los hogares); otros motivadores expresados son: festividades / temporalidad (enfoques conductista, humanista, social-cultural y postmoderno).

\section{¿En este hogar existe lealtad a más de una marca del mismo producto? ¿Por qué?}

Se observó que los cambios de marca culminan con su reconocimiento por parte de los integrantes de la familia, algunas de ellas después de la prueba (muchas veces obligada, como es el caso del desabasto) terminan siendo valoradas como buenos sustitutos por lo que son incorporadas al grupo primario de marcas del hogar; se construye así una duplicidad de marca que con el tiempo se convierte en lealtad compartida.

Los principales argumentos que justifican la duplicidad de marca son los siguientes:

- "En productos de belleza y cuidado personal, como es el caso de los desodorantes, cada integrante del hogar procura su marca" (30\% de los hogares), el motivador central es el hecho de que cada uno de los integrantes de la familia (particularmente los jóvenes), tiene necesidades, 
gustos y/o preferencias específicas, diferentes a los demás (enfoques psicodinámico, cognitivo y postmoderno).

- "Son marcas similares que en nuestra casa tienen usos diferentes" (25\% de los hogares); otros motivadores expresados son: beneficios diferentes / desabasto / una por precio y otra por calidad / funcionalidades y características similares (enfoques económico, psicodinámico, conductista y cognitivo).

- "Me gusta estar segura de tener en casa el producto cuando lo necesite" (21,7\% de los hogares), otro motivador expresado es el reconocimiento de que se trata de marcas de prestigio que poseen atributos y funciones similares (enfoques conductista, cognitivo y humanista).
Cuando se realiza una compra, ¿lo hacen considerando varios factores?, ¿cuáles?

Funcionalidad (21,7\%), Costo-Beneficio (28,3\%), Publicidad (16,7\%), Impulso (6,7\%), Punto de venta (8,3\%) Precio y Promociones (28,3\%) Recomendaciones y reconocimiento social (25,0\%); en esta relación de motivadores están representados todos los enfoques de la teoría de la motivación de compra.

\section{¿Qué persona realiza la mayor parte de las compras en el hogar?}

La tabla 5 resume las ocasiones de compra y la proporción de participación de los integrantes de las familias panelistas estudiadas, en donde se destaca que las compras son realizadas por la mamá, en las tiendas de abarrotes. 
Tabla 5. Ocasiones de compra

\begin{tabular}{|r|c|c|c|c|c|c|}
\hline Formato de tienda & Ocasiones & Mamá & Papá & Hijos & Hijas & Otros \\
\hline Tianguis & 22 & $59.1 \%$ & $0.0 \%$ & $0.0 \%$ & $40.9 \%$ & $0.0 \%$ \\
\hline Tiendas de abarrotes & 575 & $56.7 \%$ & $6.3 \%$ & $17.4 \%$ & $17.0 \%$ & $2.6 \%$ \\
\hline Tiendas de Descuento & 266 & $72.9 \%$ & $5.3 \%$ & $8.6 \%$ & $9.0 \%$ & $4.1 \%$ \\
\hline Cadenas de Conveniencia & 46 & $58.7 \%$ & $6.5 \%$ & $15.2 \%$ & $17.4 \%$ & $2.2 \%$ \\
\hline Tiendas de Autoservicio & 293 & $59.7 \%$ & $8.5 \%$ & $4.1 \%$ & $17.1 \%$ & $10.6 \%$ \\
\hline Tiendas de Membresía & 41 & $58.5 \%$ & $36.6 \%$ & $0.0 \%$ & $2.4 \%$ & $2.4 \%$ \\
\hline Autoservicios de Imágen & 34 & $55.9 \%$ & $2.9 \%$ & $5.9 \%$ & $5.9 \%$ & $29.4 \%$ \\
\hline Suma y promedios & 1,277 & $60.9 \%$ & $7.4 \%$ & $11.3 \%$ & $15.0 \%$ & $5.4 \%$ \\
\hline
\end{tabular}

Fuente: Elaboración propia ${ }^{3}$

\section{Conclusiones}

Los motivos de compra manifestados en el estudio cualitativo muestran que no existen un patrón de consumo que pueda ser expresado por un modelo/escuela específica; sin embargo, encontramos que tres escuelas se presentan con mayor frecuencia como razón que justifica la compra: en primer lugar, se identifica a la escuela conductista, las respuestas obtenidas sobre los patrones de conducta de compra nos dejan ver que estos se repiten a partir de la elección heredada, la satisfacción con el producto, el cumplimiento de expectativas y la satisfacción de necesidades; la escuela cognitiva sigue a la conductista, se observó que los miembros de la familia realizan compras repetidas en función de la experiencia de compra adquirida, misma que influye en sus futuras decisiones; la tercera escuela es la económica, las principales motivaciones de cambio de marca son el costo-beneficio, el precio y las promociones de venta que ofrecen las marcas sucedáneas, pero se encontró que este factor es temporal y luego se regresa a la marca habitual; por lo que en respuesta a nuestra hipótesis: “a pesar de que los productos de consumo recurrente siguen una tendencia sistemática de compra, los motivos de naturaleza intencionada y determinista que subyacen a la compra repetitiva, la duplicidad y sustitución de marca, la cuota de cartera y la lealtad compartida, no se identifican en

3 Mayor detalle puede ser consultado en el tercer reporte de esta investigación titulado: "Comportamientos de lealtad al punto de venta en hogares de la Ciudad de México" (Fischer, L., Espejo, J, 2016c) 
forma exclusiva con alguno de los enfoques, escuelas o teorías de la motivación de compra", encontramos que las escuelas conductista, cognitiva y económica son aquellas que se presentan como motivos de compra en un mayor número de ocasiones en los productos de consumo habitual.

La conjunción de los hallazgos cualitativos y cuantitativos de esta investigación permiten concluir que el compromiso psicológico traducido como actitud positiva de lealtad a la marca e intención de recompra, finalmente se manifiesta como recompra persistente de la marca a lo largo del tiempo.

\section{Referencias}

AMAI. (7 de 12 de 2015). Asociación Mexicana de Agencias de Investigación de Mercado y Opinión Pública. Recuperado de AMAI: http://nse.amai. org/nseamai2/

Bower, G. H. (1981). Theories of learning. Englewood Cliffs, NJ: Prentice-Hall.

Bray, J. P. (2008). Consumer Behaviour Theory: Approaches and Models. United Kingdom: Bournemouth University.
Ertmer, P. N. (1993). Conductismo, cognitivismo y constructivismo: una comparación de los aspectos críticos desde la perspectiva del diseño de instrucción. Performance improvement quarterly, 6(4), 50-72.

Fischer, L. E. (2011). Mercadotecnia. México: McGraw-Hill.

Fischer, L. E. (2015). Razones que impulsan la motivación de compra de los estudiantes universitarios mexicanos. México: FCA UNAM - UAEM.

Fischer, L., Espejo, J. (2016b). Generalizaciones en la Compra repetida, Duplicidad de marca, Sustitución de marca, Lealtad compartida entre marcas y Doble riesgo en hogares de la Ciudad de México. Puebla, México: IEU.

Fischer, L., Espejo, J. (2016c). Comportamientos de lealtad al punto de venta en hogares de la Ciudad de México. Puebla, México: IEU.

Fischer, L., Espejo, J. (2016a). Comportamientos de lealtad a la marca en hogares de la Ciudad de México. Puebla, México: IEU.

Foxall, G. (1990). Consumer psychology in behavioural perspective. London: Routledge. 
Hoyer, W. M. (2010). Comportamiento del consumidor. México: Cengage Learning.

INEGI. (2011). Panorama socioeconómico del Distrito Federal. México: Instituto Nacional de Estadística y Geografía.

McLeod, S. (2013). Psychology Behaviorist Approach. Recuperado de www.simplypsychology.org/behaviorism.html

Petrauskaite, E. (2014). Effect of brand image on consumer purchase behaviour: International footwear market comparison. Aalborg, Dinamarca: Aalborg University.

Romo, H. (2009). Niveles Socioeconómico AMAI. México: Instituto de Investigaciones Sociales SC.
Schiffman, L. K. (2007). Consumer Behavior, 9/E. New York: Prentice Hall.

Sheth, J. N. (1991). Why we buy what we buy: A theory of consumption values. Journal of Business Research, 22, 159-170.

Simon, H. (1997). Administrative behavior: A study of decision-making processes in administrative organizations, 4th Edition. New York: The Free Press.

Stewart, J. 1. (1994). Psychology of decision making. In: D. Jennings \& S. London: Wattam/Pitman. Decision Making: an Integrated Approach.

Usín, S. (2013). Experiencia de compra de los consumidores de centros comerciales en Vizcaya. Leioa: Universidad del País Vasco. 


\section{Anexo 1.}

\section{Instrumento de investigación:}

Diario de Compras

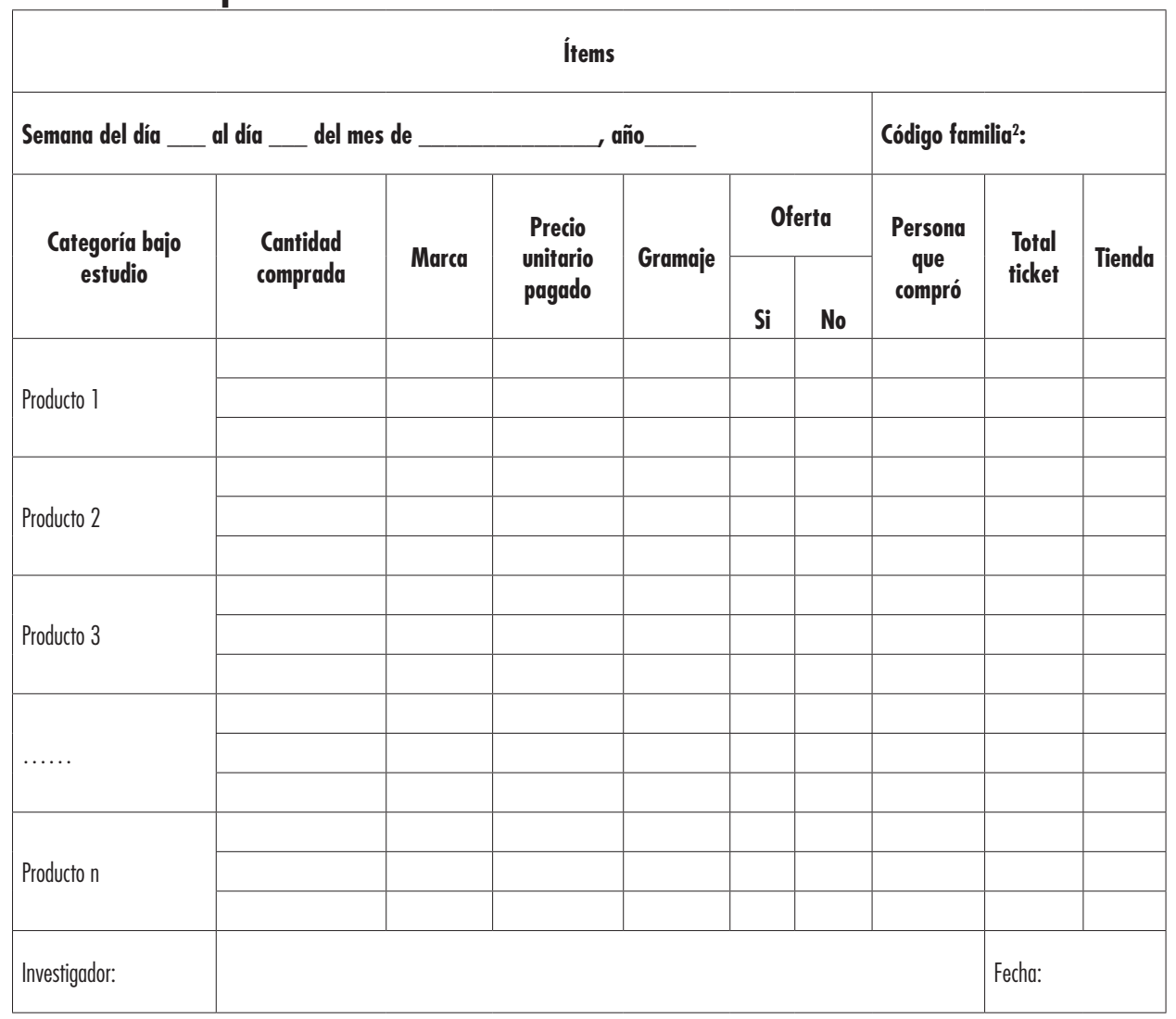

Fuente: Elaboración propia 


\section{Anexo 2. Datos de perfil de la familia panelista}

\begin{tabular}{|l|l|l|l|l|l|l|l|}
\hline Familia & $\begin{array}{l}\text { Integrantes } \\
\text { (número) }\end{array}$ & $\begin{array}{l}\text { Niños } \\
\text { (número) }\end{array}$ & $\begin{array}{l}\text { Adultos } \\
\text { mayores } \\
\text { (número) }\end{array}$ & $\begin{array}{l}\text { Mascotas } \\
\text { Si / No }\end{array}$ & $\begin{array}{l}\text { Nivel } \\
\text { educativo }\end{array}$ & $\begin{array}{l}\text { Rango de } \\
\text { Ingresos } \\
\text { (miles) }\end{array}$ & $\begin{array}{l}\text { Código } \\
\text { postal }\end{array}$ \\
\hline 1 & & & & & & & \\
\hline 2 & & & & & & & \\
\hline 3 & & & & & & & \\
\hline 4 & & & & & & & \\
\hline 5 & & & & & & & \\
\hline$\ldots$ & & & & & & & \\
\hline $\mathrm{N}$ & & & & & & & \\
\hline
\end{tabular}

Fuente: Elaboración propia

\section{Anexo 3. Instrumento de investigación: Guía de tópicos. Fuente: Elaboración propia}

1. Bienvenida y presentación

- Hola, buenos días (tardes, noches), les agradezco su presencia, lo que realizaremos a continuación se conoce como sesión de grupo y consiste en una reunión de varias personas para platicar sobre un tema en específico.

- En esta ocasión platicaremos sobre sus experiencias en el registro de las compras que reportaron como hogar panelista durante los últimos 4 meses, es importante señalar que no existen respuestas correctas o incorrectas, por lo tanto, siéntanse con toda libertad de opinar todas sus percepciones o apreciaciones.

- Les voy a pedir que hablen en un tono adecuado para que se pueda escuchar y que por favor no se interrumpan, por lo que una persona debe hablar a la vez, también es importante que apaguen sus teléfonos celulares, la sesión se grabará para no perder detalle de los comentarios.

- ¿Tienen alguna pregunta? comencemos entonces...

2. Sesión de respuestas y comentarios a las siguientes preguntas 
a. ¿Por qué en este hogar se consumen las mismas marcas?

b. ¿Por qué en este hogar se llega a cambiar de marca?

c. ¿En este hogar existe lealtad a más de una marca del mismo producto? ¿por qué?

d. Cuando se realiza una compra. ¿lo hacen considerando varios factores?, ¿cuáles?

e. ¿Qué persona realiza la mayor parte de las compras en el hogar? 